International Journal of Engineering \& Technology, $7(4.5)(2018) 313-315$
International Journal of Engineering \& Technology
SPC
Website: www.sciencepubco.com/index.php/IJET
Research paper

\title{
Study of PVDF Based Electrode Structure in Supercapacitors
}

\author{
Samata Parulekar $^{1}$,Shahbaz Sholapure ${ }^{2}$ R.M Holmukhe ${ }^{3}$, P.B. Karandikar ${ }^{4}$ \\ Research Assistant ${ }^{1}$,Associate Professor ${ }^{3}$, Department of Electrical Engineering, \\ Bharati Vidyapeeth Deemed University College of Engineering, Pune, India ${ }^{1,3}$ \\ Research Assistant ${ }^{2}$, Department of Mechanical Engineering, College of Engineering Pune, India ${ }^{2}$ \\ Associate Professor.,Department of Electronics and Telecommunication Engineering, Army Institute of Technology,Pune, India ${ }^{4}$

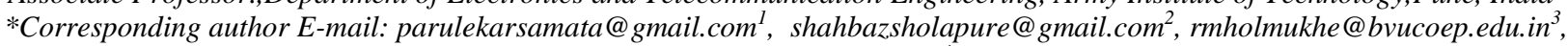 \\ pbkarandikar@gmail.com ${ }^{4}$
}

\begin{abstract}
Because of increasing demand there is a need for energy storage. Supercapacitor the new age energy storage device which can provide quick burst of energy. The study focuses on use of Polyvinylidene fluoride as the binder material in making of electrodes for supercapacitors. Polyvinylidene fluoride (PVDF) is a highly non-reactive thermoplastic fluoropolymer produced by the polymerization of vinylidene difluoride. It is a strong piezoelectric element. Piezoelectric effect is useful for generation of high voltages. Binder materials are responsible for holding the active material particles within the electrode. The study was focused on use of PVDF in Electric Double Layer Capacitors (EDLC) and Pseudo capacitors. In case of Electric Double Layer Capacitors the PVDF is used as binder material with activated carbon as electrode material. In case of Pseudo capacitor the PVDF is used as a binder material with activated carbon and metal oxide as electrode material. Research was performed with preparation of electrodes using 6 types of Activated carbons namely Vulcun XC 72, Pica, RP20, C60, NORIT and Graphene for different compositions. The test was conducted on single electrode and it was initially charged at a voltage of $2.2 \mathrm{~V}$ and its discharge was recorded for a time period of 3 minutes. The research focuses on the material which is giving the best results in regarding to the key parameters of supercapacitors and also concentrates on the composition of Polyvinylidene fluoride (PVDF) which gives the highest value of capacitance with the lowest value of Equivalent series resistance in making electrodes for supercapacitors.
\end{abstract}

Keywords: Capacitance; activated carbon; Equivalent series resistance; binder material; piezoelectric.

\section{Introduction}

Energy storage is the key component for creating sustainable energy storage systems. Energy storage technologies have the potential to offset the sporadic problem of renewable energy sources by storing the generated intermittent energy and the making it accessible upon demand. Current technologies such as solar photovoltaic's and wind turbines can generate energy in a sustainable environment friendly manner; yet their intermittent nature still prevent them from becoming the primary energy carrier. Currently the dominating energy storage device remains the battery particularly lithium ion batteries but the problem with the batteries are they store energy electrochemically that means with the passage of time the storage capacity will decrease hence resulting in low cycle life [1].This problem is addressed by supercapacitors which uses different storage mechanism. In supercapacitors energy stored electro-statically that is on the surface of material and does not involve the chemical reactions. This is the reason binder material and electrode material plays an important role in supercapacitors. Presently supercapacitors face the problem of low energy density because of poor material composition. The Supercapacitors as an energy storage device provides high power with quick burst of time and magnificent cyclic stability. Hence supercapacitors found in applications requires many rapid charge and discharge cycles. Supercapacitors found its application on various electrical vehicles as a reserve for batteries; it is also used as power provider to gazettes which needs quick burst of energy such as camera flash which requires sufficient energy of light to focus under specific area in small period of time. Normally electrodes are fabricated by brushing the slurry on the metal substrate. The slurry is prepared by stirring the mixture of active material, binder and conducting agent together. The property of binder is to avoid the active material from falling off during working of electrode, increases the mechanical strength of electrode and pore size respectively. The use of binder enhances the electrochemical performance of supercapacitors. PVDF is used as binder material in making electrodes which has properties of high thermal stability, high dielectric constant, and excellent mechanical strength. Polyvinylidine difluoride is highly non reactive thermoplastic fluoropolymer produced by polymerization of vinylidene difluoride [2]

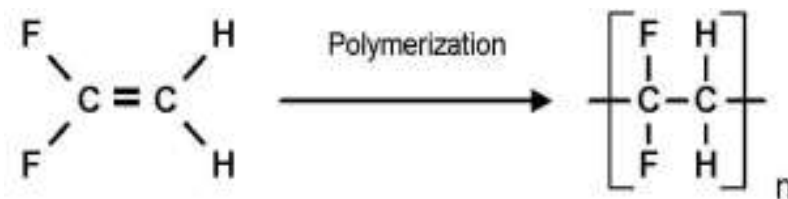

Vinylidene fluoride

PVDF

Fig.1: Chemical structure of Polyvinylidene fluoride

The electrodes play the key role in efficiency of supercapacitors. Wrong selection of electrode material or wrong composition of electrode and binder material often affects the key parameters of supercapacitors. Hence it's important to find the correct composition as well as correct electrode material for preparation of supercapacitors. The correct composition can be given mathematically as there is linear relationship between efficiency of electrode ma- 
terial and capacitance of supercapacitors.

\section{Experimentation and Results}

\subsection{Experimentation:}

The electrodes are prepared by brushing the slurry on wire mesh. The slurry preparation is different for two types of supercapacitors.

\subsubsection{Electric Double Layer Capacitors:}

Different composition of Polyvinylidene Fluoride (PVDF) is dissolved in dimethyl sulphoxide (DMSO) solvent and then in this mixture activated carbon is added. Again solvent is added in to the mixture to bring honey like texture.

\subsubsection{Pseudo capacitors:}

Different composition of Polyvinylidene Fluoride (PVDF) is dissolved in dimethyl sulphoxide (DMSO) solvent and then in this mixture activated carbon and metal oxide is added. Again solvent is added in to the mixture to bring honey like texture.

The prepared electrodes were heated at a temperature of 200 to 300 degree Celsius in a muffle furnace for about $45 \mathrm{~min}$. Then these electrodes were immersed in aqueous electrolyte which is made by using Potassium sulphate (K2SO4) and the electrodes were tested at the voltage of $2.2 \mathrm{~V}$ and discharge current is noted for 3 minutes.

\subsection{Results:}

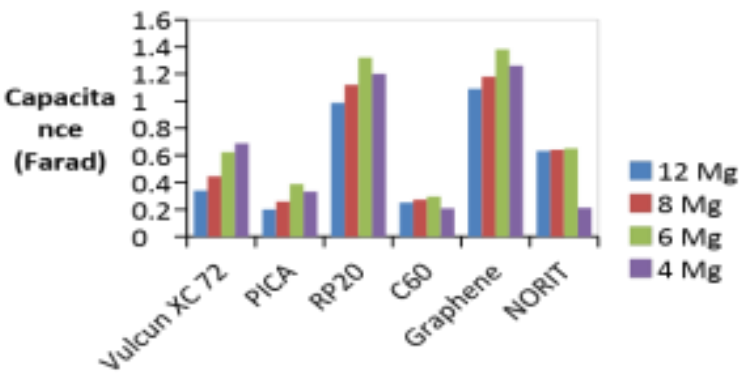

Types of Carbon Material

Fig.2: Comparison of Capacitance for various types of activated carbon materials with different Composition (For EDLC).

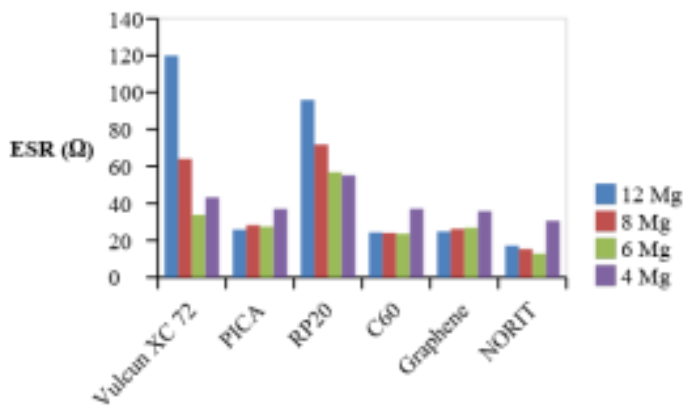

Types of Activated Carben Material

Fig.3: Comparison of Capacitance for various types of activated carbon materials with different Composition (For EDLC).

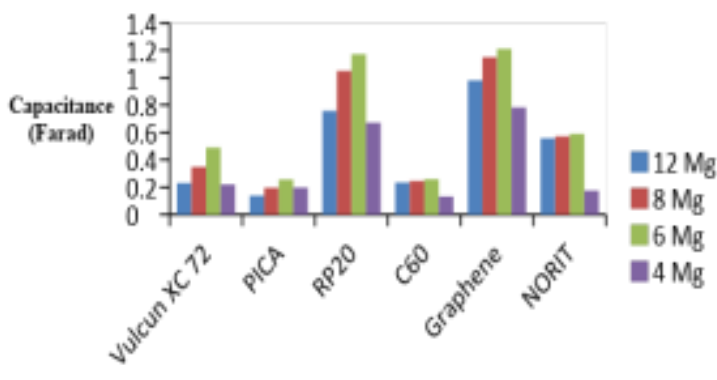

Types of Activated carbon Material

Fig.4: Comparison of capacitance for various types of activated carbon materials with different Composition (For pseudo capacitors)

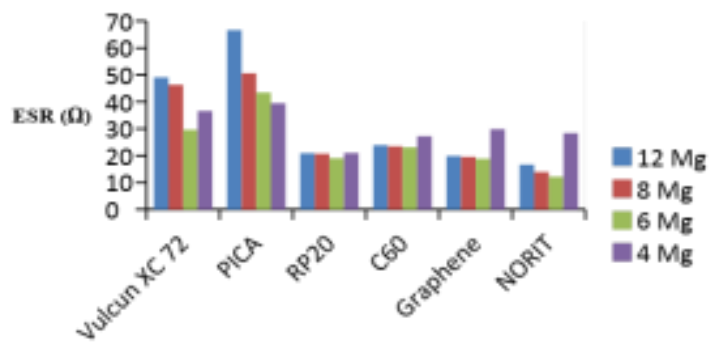

Types of Activated Carbon Material

Fig.5: Comparison of ESR for various types of activated carbon materials with different Composition (For Pseudo capacitors)

\subsection{Mathematical Equation:}

A mathematical proportion can be formulated as to how much PVDF should be taken for total slurry of PVDF and activated carbon, through this study the lab experiments have shown $6 \mathrm{mg}$ PVDF material used as binder material given the best results in case of capacitance and equivalent series resistance when total slurry of activated carbon and PVDF was $240 \mathrm{mg}$ hence it can be said in case of $6 \mathrm{mg}$

$\mathrm{PVDF}=1 / 40 \mathrm{x}$

$1 / 40$ is the multiplying factor which has given the best result in this experiment and $x$ represent the total slurry of PVDF and activated carbon used.

\section{Conclusion}

The presented work focused on the problem of poor composition and choice of electrode material. The experiment shows the importance of composition of electrode and binder materials used in making of supercapacitors. The work also reflected the importance of PVDF as a binder material coupled with the electrode material to enhance the key parameters of supercapacitors. The PVDF as a binder material prevents the activated carbon material from falling off during the discharging process of supercapacitor. The work gives an insight on mathematical equation for the composition of PVDF to be used with electrode materials in the preparation of supercapacitors. The lab results showed RP-20 and Graphene gives best results in case of key parameters of supercapacitors. This work also shows that $6 \mathrm{mg}$ composition of PVDF gives the best results in terms of capacitance and equivalent series resistance of supercapacitors. .PVDF is known as a polymeric binder, PVDF when mixed with aqueous solution forms a latex type formation due to which activated carbons stick to it during discharge process hence leading to less contact area between binder material and electrode material resulting in less internal resistance. When PVDF is used as binder material with Mno2 in Pseudo capacitors the results are better as compared to Electric Double Lay- 
er Supercapacitors. Use of PVDF causes improved electrochemical performance which can be attributed to the formation of chemical bonds that leads to rapid electron transfer and low charge transfer resistance. presently adhesive are used as binder material which increases the contact area between electrode material and binder material hence this study also provides a novel idea of using PVDF as binder material to reduce the ESR in supercapacitors.

\section{References}

[1] Marco A. Spreafico, Paula Cojocaru, Luca Magagnin, Francesco Triulzi and Marco Apostlo, "PVDF Latex As a Binder for Positive Electrodes in Lithium Ion Batteries", Vol. 53, Issue. 22: pages 9094-9100, Publication Date February 18, 2014

[2] www.targray.com, Online, Accessed 23 November 2017, www.targray.com/Li-ion-battery/binders/pvdf

[3] Viacheslav Barsykov, Volodymyr Khomenko,"The Influence of Polymer Binders on the Performance of Cathodes for Lithium Ion Batteries", unpublished

[4] E. Markevich, G. Salitra, D. Aurbach, "Influence of the PVDF binder on the stability of $\mathrm{LiCoO} 2$ electrodes", Electrochemistry Communications, Volume 7, issue 12, December 2005, Pages 1298-1304.

[5] M. Mancini, R. Marassi, "High performance, environmentally friendly and low cost anodes for lithium-ion battery based on $\mathrm{TiO} 2$ anatase and water soluble binder carboxymethyl cellulose", Journal of Power Sources, Volume 196, Issue 22, 15 November 2011, Pages 9665-9671

[6] P.B Karandikar, Dhananjay BaluTalange, Uday PrakashraoMhaskar, Ramesh Bansal, Development, Modeling and Characterization of aqueous metal oxide based supercapacitor, Exergy, Vol. 40, Issue 1, April 2012.

[7] P.B Karandikar, Dhanraj Rathod, DhananjayTalange, Photovoltaic Chargeable Electrochemical Double Layer Capacitor, Computer and Electrical Engineering, 2009. ICCEE '09. Second International Conference, 28-30 Dec. 2009

[8] L.S. Godse, P.B. Karandikar, M.Y. Khaladkar, Study of Carbon Materials and Effect of its Ball Milling, on Capacitance of Supercapacitor, Energy Procedia, Volume 54, 2014, Pages 302-309, 2014

[9] Gupta, V., and N. Miura. 2005. Electrochemically deposited polyaniline nanowire network: a high-performance electrode material for redox supercapacitor. Electrochem. Solid-State Lett. 8:A630 A632.

[10] Difference between capacitor and supercapacitor [online] Available http:// www.differencebetween.com

[11] [11] Simple capacitors to supercapacitors- An overview [online] available: http:// www.electrochemsci.org/papers/vol3/3111196

[12] P B Karandikar, Akanksh Dixit, Shailendra, Amal Paul, "Optimization of electrode parameters of stacked structure ultracapacitor" Fourth International conference on Advances in Energy Research at IITB, Mumbai, December 2013, PP.135-136

[13] Liangbing Hu, Hui Wu and Yi Cui, "Printed Energy Storage Devices by Integration of electrodes and Separators into Single Sheet of Paper.

[14] Zhentao Zhu et.al, "Effects of Various Binders on Supercapacitor Performances", "International Journal of ELECTROCHEMICAL SCIENCE, Vol. 11, Published on 06 September 2019, Pages 82708279 .

[15] J. Vetter, P. Novák, M.R. Wagner, C. Veit, K.C. Möller, J.O. Besenhard, A. Hammouche, J. Power Sources, 147 (2005) 269

[16] C.K. Subramaniam, C.S. Ramya, K. Ramya, J. Applied Electrochem, 41 (2011) 197.

[17] J. Dong, Z. Wang, X. Kang, Colloids and Surfaces A: Physicochem. Eng. Aspects, 489 (2016) 282

[18] T. Takahashi, M.I. Tejedor-Tejedor, J.J. Wouters, R. Perez-Roa, M. A. Anderson, J. Energy Storage, 7 (2016) 147.

[19] Conway, B. E. (1999). Electrochemical Supercapacitors: Scientific Fundamentals and Technological Applications. New York, KluwerPlenum. 